University of Montana

ScholarWorks at University of Montana

$3-27-2007$

\title{
Size Dependence of Static and Dynamic Magnetic Properties in Nanoscale Square Permalloy Antidot Arrays
}

\author{
Minghui Yu \\ University of New Orleans \\ Leszek M. Malkinski \\ University of New Orleans \\ Leonard Spinu \\ University of New Orleans \\ Weilie Zhou \\ University of New Orleans \\ Scott L. Whittenburg \\ University of Montana - Missoula, scott.whittenburg@umontana.edu \\ Follow this and additional works at: https://scholarworks.umt.edu/chem_pubs \\ Part of the Biochemistry Commons, Chemistry Commons, and the Physics Commons \\ Let us know how access to this document benefits you.
}

\section{Recommended Citation}

Yu, Minghui; Malkinski, Leszek M.; Spinu, Leonard; Zhou, Weilie; and Whittenburg, Scott L., "Size Dependence of Static and Dynamic Magnetic Properties in Nanoscale Square Permalloy Antidot Arrays" (2007). Chemistry and Biochemistry Faculty Publications. 71.

https://scholarworks.umt.edu/chem_pubs/71

This Article is brought to you for free and open access by the Chemistry and Biochemistry at ScholarWorks at University of Montana. It has been accepted for inclusion in Chemistry and Biochemistry Faculty Publications by an authorized administrator of ScholarWorks at University of Montana. For more information, please contact scholarworks@mso.umt.edu. 


\title{
Size dependence of static and dynamic magnetic properties in nanoscale square Permalloy antidot arrays
}

\author{
Minghui Yu, ${ }^{\text {a) }}$ Leszek Malkinski, Leonard Spinu, Weilie Zhou, and Scott Whittenburg \\ Advanced Materials Research Institute, University of New Orleans, New Orleans, Louisiana 70148
}

\begin{abstract}
(Presented on 9 January 2007; received 31 October 2006; accepted 22 November 2006; published online 27 March 2007)

Permalloy antidot arrays with different square hole sizes $(1200 \times 1200,800 \times 800$, and 400 $\times 400 \mathrm{~nm}^{2}$ ) have been fabricated by means of electron-beam lithography and lift-off techniques. The smaller square hole size results in enhanced remanence and reduced coercivity in the antidot array. Multiple resonance modes were clearly observed for the magnetic field applied normal to the array plane, and double uniform resonance modes occurred when the field deviated more than $30^{\circ}$ from the normal to the plane. Two distinct dipolar field patterns with different orientations and magnitudes split the uniform resonance into double resonance modes. The double resonance modes show uniaxial in-plane anisotropy and the easy axes are orthogonal. The magnitude of the induced dipolar anisotropy remains almost constant with changes in the square hole size. The double resonance peaks move to low field with reduction of the square hole size. (C) 2007 American Institute of Physics. [DOI: 10.1063/1.2709501]
\end{abstract}

Arrays of antidot have attracted extensive attention due to their potential application for ultrahigh density data storage. ${ }^{1,2}$ It was proposed that the memory bit could be trapped between consecutive holes along the intrinsic hard axis of the antidot nanostructure. ${ }^{2}$ One advantage of antidots over dots is that they can overcome the superparamagnetism limitation of isolated magnetic dots while preserving the properties of the magnetic film. This makes antidots a promising candidate for ultrahigh density data storage. Recently, a number of publications reported the magnetic reversal and magnetotransport properties in the antidot arrays. ${ }^{3-5}$ In contrast, relatively few investigations have been devoted to study dynamic magnetic property in antidot arrays, especially those with characterized size near several hundreds of nanometers. Yu et al. studied the ferromagnetic resonances (FMRs) in micron-sized square and rectangular Permalloy antidot arrays with circular hole size around $1.5 \mu \mathrm{m}$ in diameter and separation from 3 to $7 \mu \mathrm{m} .{ }^{6,7}$ All the square and rectangular antidot arrays show double resonances with uniaxial in-plane anisotropy, which are the consequence of a dipolar field distribution producing two regions with different demagnetization field patterns. ${ }^{6}$ Moreover, lateral spin wave modes were observed in the antidot arrays beside the main uniform mode when the magnetic field is along the film normal, which were attributed to lateral confinement from the vacant holes. ${ }^{7}$ In our previous report, we have prepared nanoscale antidot arrays on top of porous AAO membrane, and interesting results have been obtained from FMR measurement. ${ }^{8}$ In this work, we have successfully fabricated lithographically patterned antidot arrays with different nanoscale sizes of square holes on top of flat $\mathrm{Si}$ substrate surface. The detailed study on the size dependences of the static and dynamic magnetic properties of these lithographi-

\footnotetext{
${ }^{\text {a) }}$ Author to whom correspondence should be addressed; FAX: (504) 2803185; electronic mail: myu1@uno.edu
}

cally patterned nanoscale antidot arrays is presented in this contribution.

The three square Permalloy antidot arrays having constant thickness of $100 \mathrm{~nm}$ were fabricated using electronbeam lithography and lift-off techniques. First, a thin layer of polymethyl methacrylate (PMMA) resist was spun onto $\mathrm{Si}$ (100) substrates, and then patterned with an LEO $1530 \mathrm{VP}$ field emission scanning electron microscopy (FESEM) system operating at $30 \mathrm{kV}$. After development, a Permalloy film with thickness of $100 \mathrm{~nm}$ was deposited using a magnetron sputtering system with research S-gun and base pressure of $2 \times 10^{-7}$ Torr. The argon pressure during deposition was 3.0 mTorr. The deposition rate was controlled by an INFICON IC 6000 quartz monitor and was kept at $0.2 \mathrm{~nm} / \mathrm{s}$. The Permalloy film was coated with a $5 \mathrm{~nm} \mathrm{Cu}$ layer to prevent oxidation. Ultrasonic assisted lift-off in acetone was used to obtain the patterned antidot arrrays. The whole antidot pattern consists of an array of $15 \times 15$ patches; the lateral size of each patterned patch is $100 \times 100 \mu \mathrm{m}^{2}$. The spacing between each patch is around $10 \mu \mathrm{m}$, and the size of the whole pattern is $1.65 \times 1.65 \mathrm{~mm}^{2}$. Magnetic properties of the films were studied using a Quantum Design MPMS XL superconducting quantum interference device (SQUID) magnetometer at $300 \mathrm{~K}$. The ferromagnetic resonance was measured at room temperature using an X-brand Bruker EMX300 electron paramagnetic resonance (EPR) spectrometer.

The SEM images of the three antidot arrays with different square hole sizes are shown in Fig. 1. Perfect square holes were achieved in the antidot arrays with hole widths of 1200 and $800 \mathrm{~nm}$. Decreasing the hole width to $400 \mathrm{~nm}$ causes the corner of the hole to be a little rounded. The spacing between the vacant holes is fixed at $400 \mathrm{~nm}$ for all the three antidot patterns. Magnetic hysteresis loops of Permalloy reference film and the antidot arrays are presented in Fig. 2. No distinguishable in-plane anisotropy could be discerned from the hysteresis loops obtained by applying the 


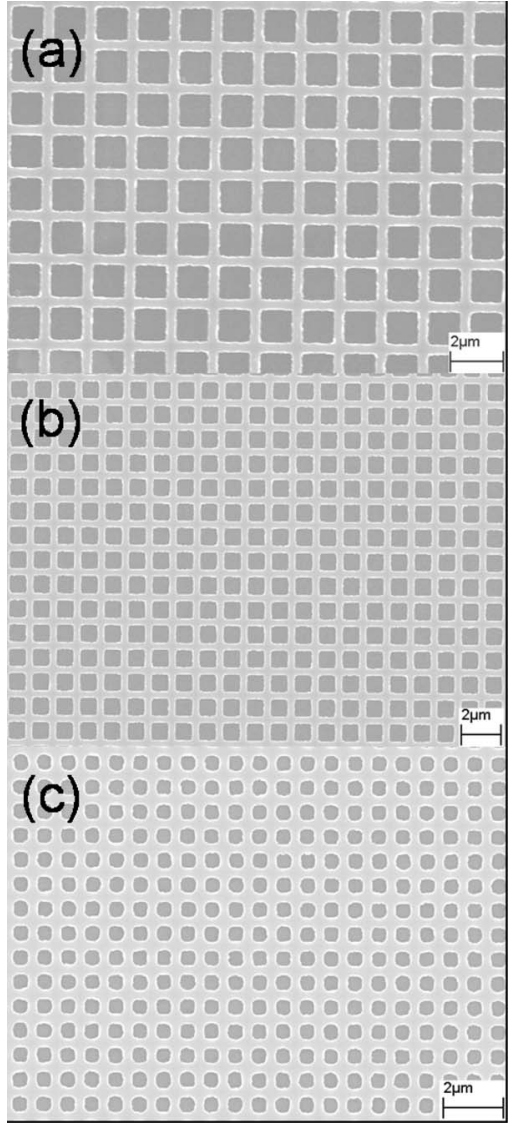

FIG. 1. SEM images of the antidot arrays with different square hole sizes: (a) $1200 \times 1200$, (b) $800 \times 800$, and (c) $400 \times 400 \mathrm{~nm}^{2}$. The separation between holes is $400 \mathrm{~nm}$.

magnetic field along different directions in the reference film plane. For the antidot arrays, the magnetic field was applied along one side of the hole. All the hysteresis loops show a gradual change in magnetization when varying the applied field. The coercivity decreases with reducing the hole size. This could be attributed to the vortex state formed around the holes; the noncollinear spin configuration around the holes promotes incoherent magnetization reversal which leads to the reduced coercivity. ${ }^{8}$ The small hole size increases the area around the holes and makes the magnetization reversal easier. For the $100 \mathrm{~nm}$ thick Permalloy antidot array formed by deposition on top of the porous AAO membrane with

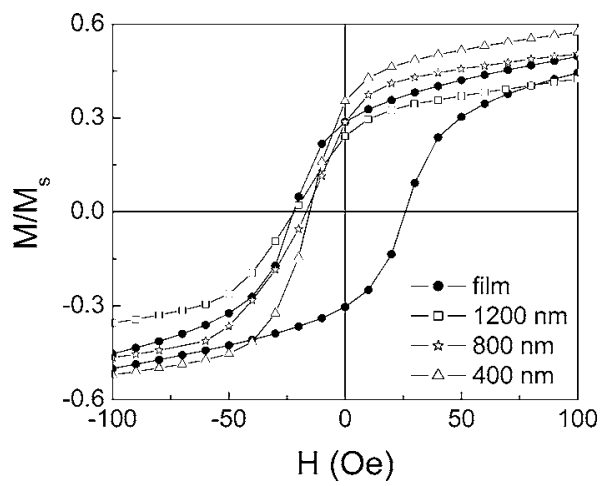

FIG. 2. Magnetic hysteresis loops of reference film and antidot arrays with different square hole widths.

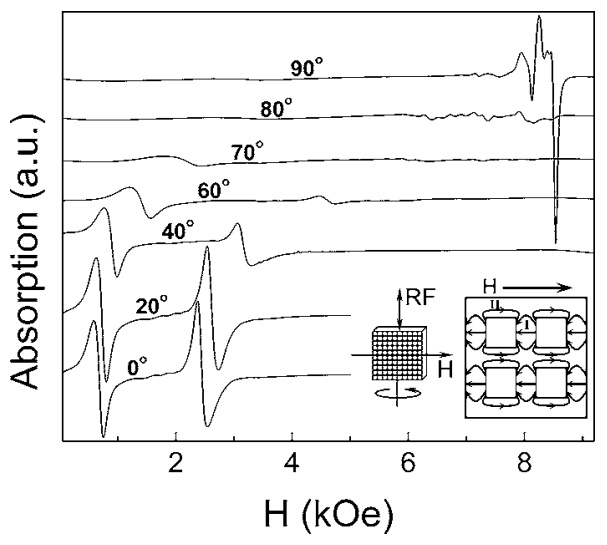

FIG. 3. FMR spectra of Permalloy antidot arrays with square hole width of $1200 \mathrm{~nm}$; out-of-plane field is applied at different angles $(\theta)$ from the array plane. The insets show the configuration of FMR measurement and the schematic demagnetization field distribution.

hexagonal arrangement of pores of $100 \mathrm{~nm}$ in diameter and spacing, a reduced coercivity was also observed in comparison with the reference film. ${ }^{8}$ The remanence first decreases for the antidot array with the $1200 \mathrm{~nm}$ hole compared with the reference film, and then increases with reducing the hole size. The demagnetization field originating from the dipoles around the sides of the holes is responsible for the smaller remanence than that of the reference film. However, the inplane dipolar interaction between the holes decreases when reducing the hole size. The reduced dipolar interaction favors alignment of the magnetizations parallel to each other and thus enhances the remanence in the antidot arrays with smaller holes.

The FMR measurements were carried out at $9.8 \mathrm{GHz}$ with the magnetic field applied both out of and in the pattern plane. The typical out-of-plane FMR spectra with the magnetic field oriented along different angles with respect to the plane were displayed in Fig. 3. When the magnetic field was along the plane normal, the sharp uniform precessional resonance mode was observed at $8485 \mathrm{Oe}$, and multiple resonance peaks also showed up at lower fields. The multiple resonance peaks are associated with the excitation of quantized standing spin wave modes due to both the perpendicular and the lateral confinements. ${ }^{7,9}$ With rotating the field away from the normal, the intensity of all the resonance peaks decreases drastically. When the field is $20^{\circ}$ away from the normal, all the spin wave modes become very vague and hardly discernible. After further rotating the field to $30^{\circ}$ away from the normal, double uniform resonance modes emerge and gradually shift to lower field with the field approaching to the plane. The double uniform resonance modes are originating from two regions experiencing different demagnetization field distributions. The inhomogeneous demagnetization field distribution was schematically illustrated in the inset of Fig. 3. When the field is applied along one side of the hole, the magnetic poles are mainly distributed around the side perpendicular to the field. Therefore, the dipolar field in region I has the opposite orientation of the external field, while that in region II has the same orientation but smaller magnitude. The distinct orientation of the dipolar fields in these two regions causes the split of the uniform 


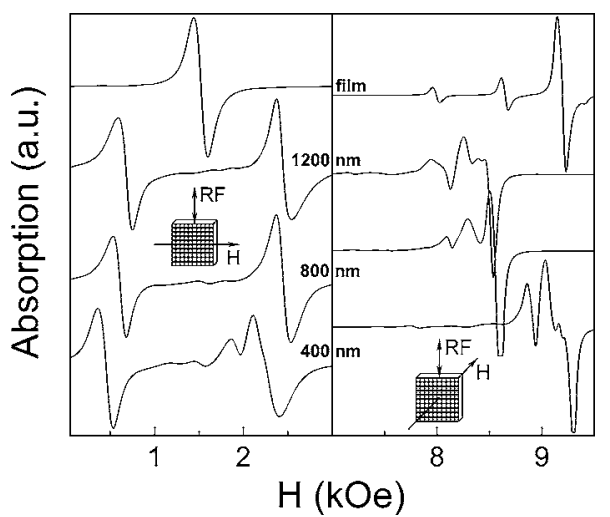

FIG. 4. FMR spectra of Permalloy reference film and square antidot arrays with different hole widths. The insets show the configuration of FMR measurement.

resonance mode into double modes. The resonance from region I shifts to higher field, while that from region II shifts to lower field. The inhomogeneous spatial distribution of the dipolar fields produced by microscale antidot arrays with circular holes has been simulated by Yu et al. ${ }^{6}$ The FMR spectra of the reference film and different antidot arrays are displayed in Fig. 4 for field in the plane (the left panel) and along the normal (the right panel). In the right panel, two well-separated standing spin wave modes were observed in the reference film at the low-field side of the uniform mode. The pinned surface-spin boundary condition or inhomogeneous internal field is responsible for two observed spin wave modes. In the antidot arrays, the uniform and spin wave modes shift to higher field with reduction of the hole size due to the enhanced out-of-plane demagnetization field. ${ }^{9,10}$ In the left panel, only the uniform mode was observed in the reference film, and the double modes move to low field with reduction of the hole size. This is due to the smaller in-plane demagnetization field in the antidot with a smaller hole size.. The two additional modes around the high-field peak in the $400 \mathrm{~nm}$ antidot array are localized edge modes, due to the sharp drop of the effective field near the hole edges. ${ }^{11}$

The in-plane angular dependences of the resonance fields of the two distinctive peaks were shown in Fig. 5. The inset shows three typical FMR spectra with field applied along the two sides of the hole and the diagonal direction. When the field is rotated away from one side of the hole, the two peaks move toward each other and merge into one peak at the diagonal direction. The single peak is closer to the low-field peak. The reason for this is that the magnitude of the dipolar field in region II is smaller than that in region I. The two peaks exhibit twofold in-plane anisotropy with orthogonal easy axes. The resonance fields have almost identical oscillatory amplitudes, which indicate good symmetry of the square hole. The resonance fields shift to lower fields with reduction of the hole size, but the oscillatory amplitudes

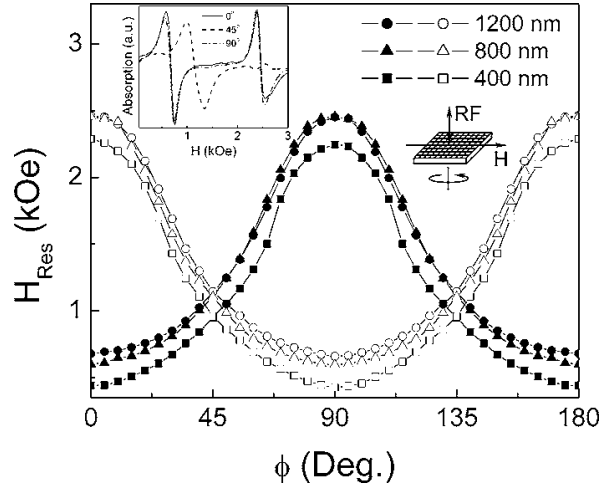

FIG. 5. In-plane angular dependence of resonance fields extracted from FMR spectra for the two distinct peaks of square antidot arrays with different hole widths. The insets show the FMR spectra of antidot array (the hole width of $1200 \mathrm{~nm}$ ) with applied in-plane field at three typical angles $\phi$ with respective to one side of the hole, and the configuration of FMR measurement.

are nearly unchanged. The magnitude of the induced dipolar anisotropy could be affected by adjusting the shape and lattice symmetry of the holes. These two distinct resonances are emanating from two different regions which experience opposite dipolar fields. The orthogonality of the easy axes and the in-plane uniaxial anisotropy indicate the uncoupled nature of these two localized resonances. ${ }^{6}$

In conclusion, the static and dynamic magnetic properties of nanoscale antidot arrays with different sizes of square holes were systematically studied. Tailoring the hole size takes effect on the remanence and the coercivity of the antidot array. The spin wave modes could be observed for the magnetic field applied along the plane normal. The inhomogeneous spatial distribution of the dipolar field splits the uniform resonance into double modes. The in-plane angular dependences of the double resonance peaks reveal the uniaxial in-plane anisotropy and orthogonality of the easy axes.

This work was supported through the National Science Foundation under NIRT grant CCF-0403673.

${ }^{1}$ L. Torres, L. Lopez-Diaz, and O. Alejos, J. Appl. Phys. 87, 5645 (2000). ${ }^{2}$ C. C. Wang, A. O. Adeyeye, and N. Singh, Nanotechnology 17, 1629 (2006).

${ }^{3}$ A. O. Adeyeye, J. A. C. Bland, and C. Daboo, Appl. Phys. Lett. 70, 3164 (1997).

${ }^{4}$ C. C. Wang, A. O. Adeyeye, N. Singh, Y. S. Huang, and Y. H. Wu, Phys. Rev. B 72, 174426 (2005).

${ }^{5}$ L. J. Heyderman et al., Phys. Rev. B 73, 214429 (2006).

${ }^{6}$ C. T. Yu, M. J. Pechan, and G. J. Mankey, Appl. Phys. Lett. 83, 3948 (2003).

${ }^{7}$ C. T. Yu, M. J. Pechan, W. A. Burgei, and G. J. Mankey, J. Appl. Phys. 95, 6648 (2004)

${ }^{8}$ A. Vovk, L. Malkinski, V. Golub, S. Whittenburg, C. O’Connor, J. S. Jung, and S. H. Min, J. Appl. Phys. 97, $10 J 506$ (2005).

${ }^{9}$ M. Nisenoff and R. W. Terhune, J. Appl. Phys. 36, 732 (1965).

${ }^{10}$ P. E. Wigen, C. F. Kooi, and M. R. Shanabarger, J. Appl. Phys. 35, 3302 (1964).

${ }^{11}$ M. J. Pechan, C. T. Yu, R. L. Compton, J. P. Park, and P. A. Crowell, J. Appl. Phys. 97, 10J903 (2005). 